\title{
$\mathrm{AIP} \mid$ chaos \\ An Interdisciplinary Journal of Nonlinear Science
}

\section{Introduction to Focus Issue: Nonlinear Dynamics for Planet Earth}

John M. Anderies, Hans G. Kaper, Emily F. Shuckburgh, and Antonios Zagaris

Citation: Chaos 25, 036201 (2015); doi: 10.1063/1.4915260

View online: http://dx.doi.org/10.1063/1.4915260

View Table of Contents: http://scitation.aip.org/content/aip/journal/chaos/25/3?ver=pdfcov

Published by the AIP Publishing

\section{Articles you may be interested in}

Introduction to Focus Issue: Statistical mechanics and billiard-type dynamical systems

Chaos 22, 026101 (2012); 10.1063/1.4730155

Introduction to Focus Issue: Nonlinear and Stochastic Physics in Biology

Chaos 21, 047501 (2011); 10.1063/1.3671647

Introduction to Focus Issue: Dynamics in Systems Biology

Chaos 20, 045101 (2010); 10.1063/1.3530126

Introduction to Focus Issue: Nonlinear Dynamics in Cognitive and Neural Systems

Chaos 19, 015101 (2009); 10.1063/1.3106111

Columbus' Earth was never FLAT

Am. J. Phys. 67, 753 (1999); 10.1119/1.19121

\section{AlP $\mid$ chaos}




\title{
Introduction to Focus Issue: Nonlinear Dynamics for Planet Earth
}

\author{
John M. Anderies, ${ }^{1, a)}$ Hans G. Kaper, ${ }^{2,3, b)}$ Emily F. Shuckburgh,, ${ }^{4,5, c)}$ \\ and Antonios Zagaris ${ }^{6, d)}$ \\ ${ }^{1}$ School of Human Evolution and Social Change, and School of Sustainability, Arizona State University, \\ P.O. Box 872402, Tempe, AZ 85287-2402, USA \\ ${ }^{2}$ Department of Mathematics and Statistics, Georgetown University, Washington, DC 20057, USA \\ ${ }^{3}$ Mathematics and Climate Research Network, 3335 Reservoir Rd, NW, Washington, DC 20007, USA \\ ${ }^{4}$ British Antarctic Survey Cambridge, CB3 OET, UK \\ ${ }^{5}$ Department of Energy and Climate Change 3 Whitehall Place, London, SWIA 2HD, UK \\ ${ }^{6}$ Department of Applied Mathematics, Universiteit Twente, 7522 NB Enschede, The Netherlands
}

(Received 6 March 2015; published online 30 March 2015)

[http://dx.doi.org/10.1063/1.4915260]

In 2013 , the community of mathematical scientists and educators focused its collective attention on the mathematics of planet Earth. In the course of the year, a grassroots organization grew into an international partnership of more than 150 scientific societies, universities, research institutes, and organizations. ${ }^{1}$ The project, known as "Mathematics of Planet Earth 2013" (MPE2013), received the patronage of UNESCO and was a truly unique event. It brought the challenges facing our planet to the attention of the mathematics research community in numerous lectures, seminars, workshops, and special sessions at conferences of the professional societies; it sponsored the development of curriculum materials for all educational levels; it organized many outreach activities, including an international juried exhibit of virtual and physical displays for use in museums and schools; and it presented a series of public lectures by renowned scientists showing the public how mathematics contributes to our understanding of planet Earth, the nature of the challenges our planet is facing, and how mathematicians contribute to their solution. At the end of the year, MPE2013 morphed into "Mathematics of Planet Earth" (MPE).

The success of MPE has led to, among other events, the establishment of new academic programs, ${ }^{2}$ the publication of two books, ${ }^{3,4}$ the initiation of new book series by commercial publishers, ${ }^{5,6}$ and the formation of a new SIAM Activity Group on Mathematics of Planet Earth by the Society of Industrial and Applied Mathematics (SIAM). ${ }^{7}$ We are extremely pleased with the initiative of AIP Publishing to devote a focus issue of CHAOS to the mathematics of planet Earth, with a special emphasis on nonlinear dynamics.

The MPE program highlighted the need for researchers with a broad view of planetary issues. Planet Earth is more than a physical system; it is also a system that supports life in all its diversity. Unfortunately, it is also a system that is increasingly being affected by human activities in ways that we are only now beginning to understand. Testing hypotheses by large-scale controlled experiments is difficult or impossible, and there is no planet B. One way to study future

\footnotetext{
${ }^{\text {a) }}$ m.anderies@asu.edu

b)hans.kaper@georgetown.edu and kaper@mathclimate.org

c)emily.shuckburgh@bas.ac.uk

d)a.zagaris@utwente.nl
}

scenarios is through large-scale computational experiments. Another, quite different approach is to focus on fundamental processes and develop mathematical models that are grounded in data but are simple enough to be effectively communicated among scholars and the public-something that benefits earth system science and policy. The latter approach, which is more conducive to mathematical analysis, can inspire new paradigms in mathematics. It was the primary motivation for the mathematical community to conceive the MPE program and turn its attention to issues of climate, global change, sustainability, biodiversity, and strategies for mitigation and adaptation.

The articles included in this focus issue illustrate the variety of nonlinear dynamics problems that arise in the study of planet Earth.

Chuter et al. ${ }^{8}$ consider a prototype dynamic vegetation box model simulating the carbon cycle of evergreen and deciduous forests. The authors find that forest survival demands a balance between foliage-allocated carbon and foliage turnover rate, as well as that two evergreen forests they examine lie close to a tipping point marking widespread mortality. They conclude their discussion by outlining the repercussions of this last finding for data assimilation.

Zhang et al. ${ }^{9}$ examine a modeling question, namely, the robustness of dispersal-induced ecological synchrony. They research multi-patch predator-prey systems, in which phaselocking between patches is due to the prey moving between patches to avoid predation. The authors find that certain modeling choices, such as saturating dispersal or accounting for finite inter-patch travel time, can effectively destroy synchrony, and thus also that the mechanism in question is not particularly robust.

Livina et al. ${ }^{10}$ outline future scenarios of atmospheric oxygen deficiency by statistically analyzing a number of oxygen time series. Tipping point analysis does not suggest the presence of critical transitions, which would carry the system into a different dynamic regime. It does predict, however, a superlinear decreasing trend in oxygen availability, which may be further exacerbated by the adoption of certain technological advances in the coming decades.

Ser-Giacomi et al. ${ }^{11}$ look at geophysical fluid circulation as a network enabling transportation, mixing, and dispersal on the planetary level. Inspired by the recent development of 
theoretical tools, they model the Mediterranean as such a large transportation network and analyze some of its properties. They also use that characterization to locate regions with strong internal and weak external mixing, knowledge of which can effectively inform environmental conservation strategies.

Ciddio et al $^{12}$ aim to understand intra- and inter-annual fluctuations in epidemic data of schistosomiasis, a parasitic disease affecting hundreds of millions of people. To that effect, they propose and analyze a dynamical model, one subunit of which describes in detail the epidemic dynamics of the disease-transmitting vector. In that manner, they identify dynamical mechanisms possibly explaining the fluctuations and predict a regime where the epidemic dynamics are chaotic.

Tantet et al. ${ }^{13}$ look for early warning signals for transitions between zonal and blocked regimes of the atmospheric circulation in the northern hemisphere, using a barotropic model of the atmosphere. Building an analytical scaffold resting on linear model reduction and transfer operators, the authors develop a probabilistic indicator, which is found to predict transitions rather accurately.

Williamson and Lenton ${ }^{14}$ also look for early warning signals for abrupt transitions in stochastically perturbed, multi-dimensional dynamical systems. Generalizing prior work, they craft statistical techniques that rely on time series analysis to differentiate between different sorts of impending transitions. In that manner, the authors develop a few simple indicators whose efficiency is illustrated on prototype, lowdimensional systems.

Doelman et al. ${ }^{15}$ highlight the importance of spatiality in ecological phenomena and develop a framework in which to analyze spatially extended systems. Specifically, they investigate consumer-resource models subject to spatial processes that play out much more slowly than the consumer's life cycle. Analyzing those, they find that such processes can destabilize emerging consumer colonies, even when the processes are passive and resource conditions favorable to growth.

Curtu and Fonley ${ }^{16}$ investigate how the possibility of flash floods is affected by rainfall history and, specifically, by the distribution of a given amount of precipitation over time. To that end, they analyze a multi-compartment model accounting for soil moisture, ground-water and surface runoff at a hillslope. In that way, they find that specific rainfall frequencies effectively prime the hillslope to a pre-flooding condition, whereas others have a much smaller effect.

Sardeshmukh and Penland ${ }^{17}$ discuss the skewed, heavytailed character of probability distributions arising in atmospheric and oceanic science. They reproduce the salient characteristics of such distributions through a stochastically forced nonlinear model whose components experience damping at disparate rates. This suggests that many characteristics of observed distributions can be attributed to interactions between strongly damped, small-scale system components with weakly damped, large-scale ones.

Siero et al. ${ }^{18}$ research a phenomenological model that describes vegetation on sloped terrains of arid ecosystems. Through nonlinear analysis and simulation, they show larger slopes to be ecologically more resilient and locate the regime in which banded vegetation transitions to dashed patterns.
Since these patterns mark a next step in the land degradation cascade, this work highlights the possibility of diversifying restoration strategies targeting arid ecosystems.

Santitissadeekorn and Jones ${ }^{19}$ identify an important problem in data assimilation and develop a method to resolve it. As they show, Kalman filter-based methods scale well with system size, but they are limited by the appearance of spurious peaks in estimated parameter distributions that adversely affect inference. To rectify this, the authors combine Kalman filtering on the system level with Bayesian techniques on the parameter one, obtaining a reliable, computationally efficient two-stage method. They demonstrate their hybrid approach by assimilating Lagrangian data into a two-layer water model.

The guest editors thank the authors for their contributions and the referees for their conscientious efforts in reviewing the manuscripts. We specially thank Kristen Overstreet and Grace Chik from the CHAOS Editorial Office, for their diligence and prompt assistance during the production of this focus Issue.

${ }^{1}$ See http://mpe2013.org for Mathematics of Planet Earth 2013.

${ }^{2}$ EPSRC Centre for Doctoral Training in Mathematics of Planet Earth (Imperial College London and University of Reading, UK); http:// mpecdt.org.

${ }^{3}$ Mathematics of Planet Earth, edited by H. Kaper and C. Rousseau (OT140, SIAM, 2015).

${ }^{4}$ Brèves de Math, Mathématiques de la planète Terre, edited by M. Andler et al. (Nouveau Monde Editions, 2014); http://www.breves-de-maths.fr.

${ }^{5}$ Mathematics of Planet Earth (Springer Verlag); http://www.springer.com/ (to be published).

${ }^{6}$ See http://degruyteropen.com for Environmental Mathematics, De Gruyter Open (DGO).

${ }^{7}$ See http://www.siam.org/activity/mpe/ for SIAM Activity Group on Mathematics of Planet Earth (SIAG/MPE), Society for Industrial and Applied Mathematics (SIAM).

${ }^{8}$ A. M. Chuter, P. J. Aston, A. Skeldon, and I. Roulstone, “A dynamical systems analysis of the data assimilation linked ecosystem carbon (DALEC) models," Chaos 25, 036401 (2015).

${ }^{9}$ Y. Zhang, F. Lutscher, and F. Guichard, "How robust is dispersal-induced spatial synchony?," Chaos 25, 036402 (2015).

${ }^{10}$ V. N. Livina, T. Vaz Martins, and A. Forbes, "Tipping point analysis of atmospheric oxygen concentration," Chaos 25, 036403 (2015).

${ }^{11}$ E. Ser-Giacomi, V. Rossi, C. Lopez, and E. Hernandez-Garcia, "Flow networks: A characterization of geophysical fluid transport," Chaos 25, 036404 (2015).

${ }^{12}$ M. Ciddio, L. Mari, M. Gatto, A. Rinaldo, and R. Casagrandi, "The temporal patterns of disease severity and prevalence in schistosomiasis," Chaos 25, 036405 (2015).

${ }^{13}$ A. Tantet, F. Van der Burgt, and H. Dijkstra, "An early warning indicator for atmospheric blocking events using transfer operators," Chaos 25, 036406 (2015).

${ }^{14} \mathrm{M}$. Williamson and T. Lenton, "Detection of bifurcations in noisy coupled systems from multiple time series," Chaos 25, 036407 (2015).

${ }^{15}$ A. Doelman, L. Sewalt, and A. Zagaris, "The effect of slow spatial processes on emerging spatiotemporal patterns," Chaos 25, 036408 (2015).

${ }^{16}$ R. Curtu and M. Fonley, "Nonlinear response in runoff magnitude to fluctuating rain patterns," Chaos 25, 036409 (2015).

${ }^{17}$ P. D. Sardeshmukh and C. Penland, "Understanding the distinctively skewed and heavy tailed character of atmospheric and oceanic probability distributions," Chaos 25, 036410 (2015).

${ }^{18}$ E. Siero, A. Doelman, M. B. Eppinga, J. D. M. Rademacher, M. Rietkerk, and K. Siteur, "Striped pattern selection by advective reaction-diffusion systems: Resilience of banded vegetation on slopes," Chaos 25, 036411 (2015).

${ }^{19}$ N. Santitissadeekorn and C. Jones, "A bimodality trap in model projections," Chaos 25, 036412 (2015). 